\title{
Global Stabilization of Discrete-Time Linear Systems Subject to Input Saturation and Time Delay
}

\author{
Xuefei Yang
}

\author{
Bin Zhou, Senior Member, IEEE,
}

Frédéric Mazenc

James Lam, Fellow, IEEE

\begin{abstract}
This paper studies the problem of global stabilization of discrete-time linear systems subject to input saturation and time delay. The considered time-delay systems are first transformed into delay-free systems based on prediction technique. Then, by utilizing saturation functions technique, the corresponding global stabilizing controllers are proposed for two special discrete-time linear systems---a chain of integrators and oscillators, and explicit conditions guaranteeing stability are also given. Both current and delayed feedback information are utilized in the controller design, and some free parameters are also introduced into these controllers. These advantages can help improve the control performance significantly. Subsequently, a systematic control design procedure for globally stabilizing general discrete-time linear systems subject to multiple inputs and/or multiple inputs delays is proposed. The design procedure is in an explicit and recursive way with explicit conditions guaranteeing stability being given, and thus is easier to use than the existing one. Finally, the effectiveness of the proposed approaches are illustrated by three numerical examples.
\end{abstract}

Index Terms-Bounded controls, Discrete-time systems, Global stabilization, Input delay, Nonlinear feedback.

\section{INTRODUCTION}

In this paper, we consider the global stabilization problem of a discrete-time linear system subject to input saturation and time delay:

$$
x(k+1)=A x(k)+B \sigma(u(k-r)), x \in \mathbf{R}^{n}, u \in \mathbf{R}^{m},
$$

where $r \geq 1$ is a known integer denoting the input delay, and $\sigma: \mathbf{R}^{m} \rightarrow \mathbf{R}^{m}$ represents actuator saturation defined as $\sigma(u)=$ $\left[\sigma\left(u_{1}\right), \sigma\left(u_{2}\right), \ldots \sigma\left(u_{m}\right)\right]^{\mathrm{T}}$ with $\sigma\left(u_{i}\right)=\operatorname{sign}\left(u_{i}\right) \min \left\{1,\left|u_{i}\right|\right\}$. Here, we have slightly abused the notation by using $\sigma$ to denote both the scalar-valued and vector-valued function. Without loss of generality, the unity saturation level was also assumed. For solving the considered problem, we also assume that the matrix pair $(A, B)$ is asymptotically null controllable by bounded controllers (ANCBC), namely, it is stabilizable in the ordinary sense and the open-loop poles are inside or on the unit circle (see, for example, [18]). It has been shown that multiple integrators with length $n \geq 3$, which is a special ANCBC system, cannot be stabilized by bounded linear feedback [11]. Thus nonlinear feedback is necessary for general ANCBC systems.

The global stabilization problem was first investigated for continuous-time systems. When the delay is absent in the control,

This work was supported in part by the National Natural Science Foundation of China (Nos. 61903102, 61773140, 61773387), the National Postdoctoral Program for Innovative Talents (No.BX20190099) and General Research Fund (No. 17200918).

Xuefei Yang is with the Department of Mechanical Engineering \& Automation, Harbin Institute of Technology(Shenzhen), Shenzhen, Guangdong, 518055, China and also with Department of Mechanical Engineering, University of Hong Kong, Hong Kong (e-mail: xfyang1989@163.com, yangxuefei@hit.edu.cn).

Bin Zhou is with the Center for Control Theory and Guidance Technology, Harbin Institute of Technology, Harbin, 150001, China (e-mail: binzhoulee@163.com, binzhou@hit.edu.cn).

Frédéric Mazenc is with EPI DISCO Inria, Laboratoire des Signaux et Systèmes (L2S, UMR CNRS 8506), CentraleSupélec, Université Paris-Sud, 3 rue Joliot Curie, 91192, Gif-sur-Yvette, France (e-mail: frederic.mazencli2s. centralesupelec.fr).

James Lam is with Department of Mechanical Engineering, University of Hong Kong, Hong Kong, China (e-mail: james. lam@hku.hk). in 1992, Teel first proposed a nonlinear controller for globally stabilizing a chain of integrators by establishing the nested saturation technique [12]. Subsequently, Sussmann et al. successfully utilized the nested saturation technique to solve the global stabilization problem of general ANCBC linear systems [10]. Since controllers designed by Teel's method often perform unsatisfactorily, a number of modifications have been made to improve the control performance (see, for example, [5], [22]). When the delay is present in the control, Mazenc et al. first extended Teel's nested saturation technique to solve the problem of global stabilization of a chain of integrators by establishing a family of nested saturation nonlinear controllers [7]. Later on, Yakoubi et al. utilized the same approach to solve the same problem for general ANCBC linear systems [15]. However, due to the existence of time delay in the input, the decoupling property in Teel's recursive design cannot be maintained in [7] and [15], which yields complex stability analyses and slightly conservative results. Recently, the same problem for multiple integrators and multiple oscillators were revisited in [23] and [17], respectively, and some families of nonlinear control laws containing both current and delayed state information were proposed based on some new special canonical forms. The approaches can take advantage of the decoupling property in the recursive design, which yields easier stability analyses and less conservative results. Teel's recursive design has also been extended to feedforward nonlinear systems subject to input saturation and/or time-delay [3], [8], [19], [20].

The global stabilization problem of discrete-time systems has also received considerable attention in the literature [6], [9], [14], [16], [18]. When the delay is absent in the control, Yang et al. in [18] studied the global stabilization of general ANCBC discrete-time linear systems by extending the results for continuous-time systems in [10], and two families of nonlinear controllers consisting of nested and cascade saturations were proposed. Probably due to the complexity of the stability analysis there, no explicit conditions guaranteeing stability were provided in [18]. Subsequently, combining the works with static saturation level in [18] and state-dependent saturation level in [5], Marchand et al. in [6] proposed a family of nonlinear controllers composed of state-dependent saturation functions for discrete-time multiple integrators. These methods were further improved in [24]. When the delay is present in the control, according to the results in [15] and [18], Yakoubi et al. given two explicit expressions of nonlinear feedbacks, including nested saturation type and cascade saturation type, for globally stabilizing general discretetime linear systems [16]. However, explicit conditions guaranteeing stability still were not given in [16]. Recently, the methods in [23] have been extended to the discrete-time multiple integrators case [24], in which two types of nonlinear controllers were proposed, and explicit conditions guaranteeing stability were also given.

In this paper, we revisit the above mentioned global stabilization problem by utilizing prediction technique and saturation functions technique. The main contribution of this paper can be stated as follows. First, we transform the considered time-delay system subject to input saturation into a delay-free system based on prediction technique, and then we design the stabilizing controller for the transformed delay-free system. Compared with the results in [16] and [24], 
the main merit of this treatment is that the destabilizing influence of time delay on the design process of controllers can be avoided, which can make the stability analysis much easier. Second, we provide explicit solutions to the above mentioned global stabilization problem based on some special canonical forms for systems characterized by both a chain of discrete-time integrators and oscillators, and also establish explicit conditions with low conservatism to guarantee the stability of closed-loop systems. Both current and delayed feedback information are utilized in the controller design, and a number of free parameters are also introduced into these controllers. These advantages can help improve the control performance significantly. Third, we provide a systematic control design procedure for globally asymptotically stabilizing general discrete-time time-delay linear systems subject to multiple inputs by combining the results for discrete-time integrators and oscillators mentioned above. Our design procedure is recursive based on explicit stability conditions given, and thus is easier to use than the existing design procedure. Finally, it is worth mentioning that the proposed approach can also be applied to solve the global stabilization problem of general discretetime linear systems with multiple input delays, which, to the best of our knowledge, seems to be an open problem.

The rest of this paper is organized as follows. The model transformation is given in Section II. The control designs of two special ANCBC linear systems including multiple integrators and multiple oscillators are given in Section III. In Section IV, we consider general discrete-time linear systems subject to input saturations and time delays. Numerical examples are given to demonstrate the effectiveness of the proposed approaches in Section V, and Section VI concludes the paper.

Notation: The notation used in this paper is fairly standard. For two integers $p$ and $q$ with $p \leq q$, the symbol $\mathbf{I}[p, q]$ refers to the set $\{p, p+1, \ldots, q\}$. For a positive constant $\varepsilon, \sigma_{\varepsilon}(x) \triangleq$ $\operatorname{sign}(x) \min \{|x|, \varepsilon\}$, particularly, $\sigma(x)=\sigma_{1}(x)$ denotes the standard saturation function. $I_{k}, k=1,2,3, \ldots$, refers to the identity matrix in $\mathbf{R}^{k \times k}$. The notation $|\cdot|$ refers to both the induced matrix 2-norm and the usual Euclidean vector norm. The notation $\otimes$ denotes the Kronecker product. For the matrix pair $(A, B)\left(A \in \mathbf{R}^{n \times n}, B \in\right.$ $\left.\mathbf{R}^{n \times m}\right), Q_{\mathrm{c}}(A, B) \triangleq\left[B, A B, \ldots, A^{n-1} B\right]$.

\section{THE MOdEL TRANSFORMATION}

In this paper, we focus on the global stabilization problem of system (1). In order to weaken the destabilizing influence of time delay in the control design, we will first transform system (1) into a delay-free system by extending the predictor-based control scheme for discrete-time time-delay linear system in [2] (see (6)-(7) there) to our cases with bounded controls, which can be described as follows.

Consider the following new state vector:

$$
y(k)=A^{r} x(k)+\sum_{i=k-r}^{k-1} A^{k-i-1} B \sigma(u(i)) .
$$

By using (2), system (1) can be expressed as

$$
y(k+1)=A y(k)+B \sigma(u(k)),
$$

which is delay-free now. The relation between systems (1) and (3) can be stated as follows: if system (3) is globally stabilized by a controller $u(k)$, then $\lim _{k \rightarrow \infty}|y(k)|=0$ and $\lim _{k \rightarrow \infty}|u(k)|=0$. From (2) we further have $\lim _{k \rightarrow \infty}|x(k)|=0$, which implies that system (1) can also be globally stabilized by the same controller $u(k)$. On the other hand, it follows from $(2)$ that $y(k)$ just requires the current information of $x(k)$ and the delayed information of $u(i), i \in \mathbf{I}[k-$ $r, k-1]$, then the controller $u(k)$ for system (1) is implementable.
Thus, it remains to design a stabilizing controller $u(k)$ for system (3).

Remark 1: For globally asymptotic stabilization of system (1), Yakoubi et al. in [16] have given two families of nonlinear controllers consisting of nested and cascade saturation functions with the aid of some canonical forms introduced by Yang et al. in [18]. However, because of the presence of delay in the control, the decoupling property in the recursive design for delay-free systems (see [12], [18]) is no longer valid, which makes the theoretical analysis in [16] rather involved. Probably due to the complexity of analysis there, in [16] the authors just proved the existence of controllers for globally stabilizing for system (1) by induction, not giving explicit conditions to guarantee the stability of the closed-loop system. In this paper, with the aid of model transformation (see (2)), we just need to consider the problem of global stabilization of delay-free system (3), which avoids the destabilizing influence of time delay in the control design, and will result in a quite easier analysis. Moreover, explicit conditions guaranteeing stability will be also given.

Remark 2: For globally asymptotically stabilizing system (3), Yang et al. in [18] also have established two families of nonlinear controllers by utilizing Teel's recursive design (see [18]). As discussed in [18], the performances of designed controllers can be divided into two parts: the first part in the nonlinear region (some saturation elements are saturated) and the second part in the linear region (none of the saturation elements is saturated). However, probably due to the complexity of trajectory analysis there, no explicit conditions were provided in [18] to guarantee the designed controllers working in the linear region. In this paper, based on some new canonical forms and by utilizing invariant set theory, we will not only give explicit conditions guaranteeing stability, but also introduce some free parameters in the controller design for improving the control performance.

\section{Two Special Linear Systems}

For convenience, in this section we first consider two special ANCBC linear systems: a chain of integrators and oscillators. Based on some special canonical forms of considered systems, globally stabilizing controllers with explicit conditions will be established.

\section{A. A Chain of Integrators}

In this subsection, we consider the following discrete-time multiple integrators system:

$$
x(k+1)=A_{\mathrm{s}} x(k)+b_{\mathrm{s}} \sigma(u(k-r)), x \in \mathbf{R}^{n}, u \in \mathbf{R},
$$

where

$$
A_{\mathrm{s}}=\left[\begin{array}{ccccc}
1 & 1 & 0 & \cdots & 0 \\
& 1 & \ddots & \ddots & \vdots \\
& & \ddots & 1 & 0 \\
& & & 1 & 1 \\
& & & & 1
\end{array}\right], b_{\mathrm{s}}=\left[\begin{array}{c}
0 \\
\vdots \\
0 \\
0 \\
1
\end{array}\right]
$$

By using the new state vector

$$
y(k)=A_{\mathrm{s}}^{r} x(k)+\sum_{i=k-r}^{k-1} A_{\mathrm{s}}^{k-i-1} b_{\mathrm{s}} \sigma(u(i)),
$$

system (4) can be rewritten as

$$
y(k+1)=A_{\mathrm{s}} y(k)+b_{\mathrm{s}} \sigma(u(k)) .
$$

Thus, as discussed in the above section, we just need to design a stabilizing controller $u(k)$ for system (7). To this end, similar to the treatment for delay-free cases in [24], the following lemma is needed. 
Lemma 1: [6] Consider the following discrete-time system:

$$
z(k+1)=A_{\mathrm{st}} z(k)+b_{\mathrm{st}} \sigma(u(k)),
$$

where

$$
A_{\mathrm{st}}=\left[\begin{array}{ccccc}
1 & \lambda_{2} & \cdots & \lambda_{n-1} & \lambda_{n} \\
& 1 & \ddots & \vdots & \vdots \\
& & \ddots & \lambda_{n-1} & \lambda_{n} \\
& & & 1 & \lambda_{n} \\
& & & & 1
\end{array}\right], \quad b_{\mathrm{st}}=\left[\begin{array}{c}
1 \\
\vdots \\
1 \\
1 \\
1
\end{array}\right]
$$

in which $\lambda_{i}, i \in \mathbf{I}[2, n]$, are a series of given non-zero numbers. Then (7) can be transformed into system (8) by the following invertible transformation:

$$
z(k)=Q_{\mathrm{c}}\left(A_{\mathrm{st}}, b_{\mathrm{st}}\right) Q_{\mathrm{c}}^{-1}\left(A_{\mathrm{s}}, b_{\mathrm{s}}\right) y(k) .
$$

It follows from Lemma 1 that system (7) is globally diffeomorphic to system (8). Thus we finally just need to design a stabilizing controller $u(k)$ for system (8). Motivated by the work in [24], we have the following theorems, whose proofs are obvious by Theorems $1--2$ in [24] and are omitted.

Theorem 1: Let $\lambda_{i}, i \in \mathbf{I}[1, n]$, be a set of given positive constants satisfying

$$
0<\lambda_{i}<1, i \in \mathbf{I}[1, n],
$$

and $\varepsilon_{i}, i \in \mathbf{I}[1, n]$, be a set of positive constants satisfying

$$
\sum_{j=1}^{i-1} \varepsilon_{j}<\varepsilon_{i}, i \in \mathbf{I}[2, n], \quad \sum_{j=1}^{n} \varepsilon_{j} \leq 1 .
$$

Then the global stabilization problem for system (4) can be solved by $u(k)=-u_{n}(k)$, in which

$$
\left\{\begin{array}{l}
u_{i}(k)=\sigma_{e_{i}(k)}\left(\lambda_{i} z_{i}(k)\right)+u_{i-1}(k), i \in \mathbf{I}[2, n], \\
u_{1}(k)=\sigma_{e_{1}(k)}\left(\lambda_{1} z_{1}(k)\right)
\end{array}\right.
$$

where $z(k)=\left[z_{1}(k), z_{2}(k), \ldots, z_{n}(k)\right]^{\mathrm{T}}$ satisfies (10) and (6), $e_{n}(k)=\varepsilon_{n}$ and $e_{i-1}(k), i \in \mathbf{I}[2, n]$, are determined by

$$
e_{i-1}(k)= \begin{cases}\varepsilon_{i-1}^{*}(k), & \text { if }\left|\lambda_{i} z_{i}(k)\right| \leq \varepsilon_{i}, \\ \varepsilon_{i-1}, & \text { if }\left|\lambda_{i} z_{i}(k)\right|>\varepsilon_{i},\end{cases}
$$

in which $\varepsilon_{i-1}^{*}(k)=\varepsilon_{i-1}+\left(e_{i}(k)-\left|\sigma_{e_{i}(k)}\left(\lambda_{i} z_{i}(k)\right)\right|\right)$.

Theorem 2: Let $\lambda_{i}, i \in \mathbf{I}[1, n]$, be a set of given positive constants satisfying (11), and $\varepsilon_{i}, i \in \mathbf{I}[1, n]$, be a set of positive constants satisfying

$$
\varepsilon_{i-1}<\frac{1}{2} \varepsilon_{i}, i \in \mathbf{I}[2, n], \quad \varepsilon_{n} \leq 1 .
$$

Then the global stabilization problem for system (4) can be solved by $u(k)=-u_{n}(k)$, in which

$$
\left\{\begin{array}{l}
u_{i}(k)=\sigma_{e_{i}(k)}\left(\lambda_{i} z_{i}(k)+u_{i-1}(k)\right), i \in \mathbf{I}[2, n], \\
u_{1}(k)=\sigma_{e_{1}(k)}\left(\lambda_{1} z_{1}(k)\right),
\end{array}\right.
$$

where $z(k)=\left[z_{1}(k), z_{2}(k), \ldots, z_{n}(k)\right]^{\mathrm{T}}$ satisfies (10) and (6), $e_{n}(k)=\varepsilon_{n}$ and $e_{i-1}(k), i \in \mathbf{I}[2, n]$, are determined by

$$
e_{i-1}(k)= \begin{cases}e_{i}(k)-\left|\lambda_{i} z_{i}(k)\right|, & \text { if }\left|\lambda_{i} z_{i}(k)\right| \leq \varepsilon_{i}-\varepsilon_{i-1} . \\ \varepsilon_{i-1}, & \text { if }\left|\lambda_{i} z_{i}(k)\right|>\varepsilon_{i}-\varepsilon_{i-1} .\end{cases}
$$

We end this section with one remark

Remark 3: Compared with the results in Theorems 3-4 in [24], the controllers proposed in Theorems 1-2 in this paper not only utilize the delayed information (see (6)) for feedback, but also introduce the so-called state-dependent saturation functions into these two types of control laws to replace the standard saturation functions.
Moreover, the ranges of parameters $\left\{\lambda_{i}, \varepsilon_{i}\right\}$ (see (11), (12) and (13)) in Theorems 1-2 cover those (see (45), (47) and (55) there) in Theorems 3-4 in [24]. These advantages can improve the transient performance of the closed-loop system significantly.

\section{B. A Chain of Oscillators}

In this subsection, we consider the following discrete-time linear system:

$$
x(k+1)=A_{\circ} x(k)+b_{\circ} \sigma(u(k-r)), x \in \mathbf{R}^{n}, u \in \mathbf{R},
$$

where $\left(A_{\circ}, b_{\circ}\right)$ is controllable and all eigenvalues of $A_{\circ}$ are in the following form:

$$
\lambda\left(A_{\circ}\right)=\alpha_{i} \pm \beta_{i} \mathrm{j}, \alpha_{i}^{2}+\beta_{i}^{2}=1, \beta_{i} \neq 0, i \in \mathbf{I}[1, p],
$$

in which $p=n / 2$. Linear systems that satisfy (16) contain multiple oscillators. In this subsection, we are interested in designing nonlinear controllers that stabilize system (15) globally. Similar to the case of multiple integrators, we first introduce the following state vector:

$$
y(k)=A_{\mathrm{o}}^{r} x(k)+\sum_{i=k-r}^{k-1} A_{\mathrm{o}}^{k-i-1} b_{\mathrm{o}} \sigma(u(i)),
$$

by which system (15) can be rewritten as

$$
y(k+1)=A_{\circ} y(k)+b_{\circ} \sigma(u(k)) .
$$

Then, we further need a special state space description of system (18), as stated in the following lemma, whose proof is placed in Appendix for sake of continuity.

Lemma 2: Consider the following discrete-time system:

$$
z(k+1)=A_{\mathrm{ot}} z(k)+b_{\mathrm{ot}} \sigma(u(k)),
$$

where

$$
A_{\mathrm{ot}}=\left[\begin{array}{ccccc}
A_{1} & -b F_{2} & \cdots & -b F_{p-1} & -b F_{p} \\
& A_{2} & \cdots & -b F_{p-1} & -b F_{p} \\
& & \ddots & \vdots & \vdots \\
& & & A_{p-1} & -b F_{p} \\
& & & & A_{p}
\end{array}\right], b_{\mathrm{ot}}=\left[\begin{array}{c}
b \\
b \\
\vdots \\
b \\
b
\end{array}\right],
$$

in which

$$
F_{i}=-\gamma_{i}\left(1+b^{\mathrm{T}} b\right)^{-1} b^{\mathrm{T}} A_{i}, i \in \mathbf{I}[1, p],
$$

and

$$
A_{i}=\left[\begin{array}{cc}
\alpha_{i} & -\beta_{i} \\
\beta_{i} & \alpha_{i}
\end{array}\right], i \in \mathbf{I}[1, p], b=\left[\begin{array}{l}
0 \\
1
\end{array}\right],
$$

where $\left\{\alpha_{i}, \beta_{i}\right\}, i \in \mathbf{I}[1, p]$, satisfy (16) and $\gamma_{i} \in(0,2], i \in \mathbf{I}[1, p]$. Then system (18) can be transformed into (19) by using the following invertible transformation

$$
z(k)=Q_{\mathrm{c}}\left(A_{\mathrm{ot}}, b_{\mathrm{ot}}\right) Q_{\mathrm{c}}^{-1}\left(A_{\mathrm{o}}, b_{\mathrm{o}}\right) y(k) .
$$

To sum up, if system (19) is globally stabilized by a controller $u(k)$, then system (15) can be also globally stabilized by the same controller $u(k)$. Thus, it remains to design a stabilizing controller $u(k)$ for system (19). To this end, we need the following technical lemma whose proof is also given in Appendix.

Lemma 3: Assume that the matrix pair $(A, B) \in$ $\left(\mathbf{R}^{n \times n}, \mathbf{R}^{n \times m}\right)$ is controllable and $A$ is orthogonal (i.e., $A^{\mathrm{T}} A=I_{n}$ ), which implies that there exists a $P>0$ satisfying

$$
A^{\mathrm{T}} P A-P=0 .
$$

Let $\gamma>0$ be an arbitrary number and define

$$
A_{\mathrm{c}}=A+B F, F=-\gamma\left(I_{n}+B^{\mathrm{T}} P B\right)^{-1} B^{\mathrm{T}} P A \text {. }
$$


Then $\left(A_{\mathrm{c}}, F\right)$ is observable. Moreover, if $\gamma \in(0,2], A_{\mathrm{c}}$ is asymptotically stable, and there exist a $P_{\mathrm{o}}>0$ and a $\lambda \in(0,1)$ such that

$$
A_{\mathrm{c}}^{\mathrm{T}} P_{\mathrm{o}} A_{\mathrm{c}} \leq \lambda^{2} P_{\mathrm{o}}
$$

Let

$$
A_{\mathrm{c} i}=A_{i}+b F_{i}, i \in \mathbf{I}[1, p],
$$

with $\left\{A_{i}, b, F_{i}\right\}$ being given by Lemma 2 . Then it follows from Lemma 3 that, there exist $P_{i}>0$ and $\lambda_{i} \in(0,1), i \in \mathbf{I}[1, p]$, satisfying Lyapunov inequalities

$$
A_{\mathrm{c} i}^{\mathrm{T}} P_{i} A_{\mathrm{c} i} \leq \lambda_{i}^{2} P_{i}, i \in \mathbf{I}[1, p] .
$$

For future use, we denote

$$
p_{i}^{-}=\lambda_{\min }\left(P_{i}\right), p_{i}^{+}=\lambda_{\max }\left(P_{i}\right), i \in \mathbf{I}[1, p] .
$$

With the aid of above preliminary results, we are ready to give the main results in this subsection.

Theorem 3: Let $\gamma_{i} \in(0,2], i \in \mathbf{I}[1, p], \lambda_{i} \in(0,1), i \in \mathbf{I}[1, p]$, and $\varepsilon_{i}, i \in \mathbf{I}[1, p]$, be a series of positive constants satisfying $\varepsilon_{p} \leq 1$ and

$$
\left(\frac{3 \gamma_{i} p_{i}^{+}}{2\left(1-\left(1+\eta_{i}\right) \lambda_{i}\right) p_{i}^{-}}+1\right) \varepsilon_{i-1}<\varepsilon_{i}, i \in \mathbf{I}[2, p],
$$

where $\eta_{i} \in\left(0,\left(1-\lambda_{i}\right) / \lambda_{i}\right), i \in \mathbf{I}[2, p]$, and $\left\{p_{i}^{-}, p_{i}^{+}\right\}, i \in \mathbf{I}[2, p]$, are given by (29). Then the global stabilization problem for system (15) is solved by $u(k)=u_{p}(k)$, in which

$$
\left\{\begin{aligned}
u_{i}(k) & =\sigma_{\varepsilon_{i}}\left(F_{i} z_{i}(k)+u_{i-1}(k)\right), i \in \mathbf{I}[2, p], \\
u_{1}(k) & =\sigma_{\varepsilon_{1}}\left(F_{1} z_{1}(k)\right),
\end{aligned}\right.
$$

where $z(k)=\left[z_{1}^{\mathrm{T}}(k), z_{2}^{\mathrm{T}}(k), \ldots, z_{p}^{\mathrm{T}}(k)\right]^{\mathrm{T}}$ with $z_{i}(k) \in \mathbf{R}^{2}, i \in$ $\mathbf{I}[1, p]$, satisfies (23) and (17).

Proof: Please refer to Appendix.

We end this section with two remarks.

Remark 4: Benefit from the design scheme for removing the saturation function in Lemma 4 (see Appendix), Teel's forwarding design (see [3], [7], [12]) can be easily extended to Theorem 3, namely, we just need to handle a planar system at each step as all other state components are canceled in the recursive design. Moreover, in the controller design, some free parameters are introduced that can be well designed to improve the control performance.

Remark 5: We give a brief discussion about the effect of free parameters on control performance. On the one hand, in order to fully utilize the control energy, it follows from (30) that the values of $E_{i} \triangleq 3 \gamma_{i} p_{i}^{+} /\left(\left(1-\left(1+\eta_{i}\right) \lambda_{i}\right) p_{i}^{-}\right), i \in \mathbf{I}[2, p]$, should be as small as possible. Since $\left\{p_{i}^{+}, p_{i}^{-}, \lambda_{i}\right\}, i \in \mathbf{I}[2, p]$, are all functions of $\gamma_{i}$ by (28), then for given $\gamma_{i}, i \in \mathbf{I}[2, p]$, we can find minimal values of $E_{i}, i \in \mathbf{I}[2, p]$, via numerical simulation. On the other hand, the trajectories of the closed-loop system can be divided into two parts: the first part in the nonlinear region and the second part in the linear region. It is well known that the transient performance of the second part depends on the pole locations of the linearized closedloop system. However, the transient performance of the first part is not easy to characterize properly due to the fact that the nonlinear region is only attractive. To the best of our knowledge, there is no existing results that can be used to deal with such a optimal problem in theory. Therefore, a trial-and-error procedure via numerical simulation seems necessary.

\section{General Linear Systems}

Now we consider the global stabilization problem of general linear system (1). Since system (1) is ANCBC, there exists a nonsingular matrix $T$ such that system (1) is transformed into the following system:

$$
\left\{\begin{array}{l}
\vartheta_{\mathrm{p}}(k+1)=A_{\mathrm{p}} \vartheta_{\mathrm{p}}(k)+B_{\mathrm{p}} \sigma(u(k-r)), \\
\vartheta_{\mathrm{q}}(k+1)=A_{\mathrm{q}} \vartheta_{\mathrm{q}}(k)+B_{\mathrm{q}} \sigma(u(k-r)),
\end{array}\right.
$$

where $\left[\vartheta_{\mathrm{p}}^{\mathrm{T}}(k), \vartheta_{\mathrm{q}}^{\mathrm{T}}(k)\right]^{\mathrm{T}}=T x(k)$ and

$$
T A T^{-1}=\left[\begin{array}{ll}
A_{\mathrm{p}} & \\
& A_{\mathrm{q}}
\end{array}\right], T B=\left[\begin{array}{c}
B_{\mathrm{p}} \\
B_{\mathrm{q}}
\end{array}\right],
$$

in which $A_{\mathrm{q}} \in \mathbf{R}^{n_{\mathrm{q}} \times n_{\mathrm{q}}}$ is asymptotically stable, all eigenvalues of $A_{\mathrm{p}} \in \mathbf{R}^{n_{\mathrm{p}} \times n_{\mathrm{p}}}$ are on the unit circle, and $n_{\mathrm{p}}+n_{\mathrm{q}}=n$. It is clear that $(A, B)$ is stabilizable if and only if $\left(A_{\mathrm{p}}, B_{\mathrm{p}}\right)$ is controllable. As $A_{\mathrm{q}}$ is asymptotically stable, the second subsystem in (32) does not affect the stabilizability of the whole system. Thus, without loss of generality, we can impose the following assumption on system (1).

Assumption 1: The matrix pair $(A, B)$ is controllable and all eigenvalues of $A$ are on the unit circle.

As discussed in Section II, system (1) can be transformed into system (3) by utilizing (2), and then it remains to design the stabilizing controller for system (3).

\section{A. Single-input Case}

Under Assumption 1, when $m=1$, by using Proposition 1 in [21], there exists a nonsingular matrix $T$ such that system (3) is transformed into the following system:

$$
z(k+1)=A_{\mathrm{t}} z(k)+B_{\mathrm{t}} \sigma(u(k)),
$$

with

$$
A_{\mathrm{t}}=\left[\begin{array}{ll}
A_{11} & A_{12} \\
& A_{22}
\end{array}\right], B_{\mathrm{t}}=\left[\begin{array}{l}
b_{01} \\
b_{02}
\end{array}\right]
$$

where $\left(A_{11}, b_{01}\right) \in\left(\mathbf{R}^{2 p_{1} \times 2 p_{1}}, \mathbf{R}^{2 p_{1} \times 1}\right)$ is in the same form of $\left(A_{\text {ot }}, b_{\text {ot }}\right)$ (see (20)) with $p=p_{1},\left(A_{22}, b_{02}\right) \in\left(\mathbf{R}^{p_{2} \times p_{2}}, \mathbf{R}^{p_{2} \times 1}\right)$ is in the same form of $\left(A_{\mathrm{st}}, b_{\mathrm{st}}\right)$ (see (9)) with $n=p_{2}, A_{12}=$ $\left[\lambda_{1}, \lambda_{2}, \ldots, \lambda_{p_{2}}\right] \otimes b_{01} \in \mathbf{R}^{2 p_{1} \times p_{2}}$, in which $2 p_{1}+p_{2}=n, \lambda_{i} \in$ $(0,1), i \in \mathbf{I}\left[1, p_{2}\right]$. As discussed above, if system (33) is globally stabilized by a controller $u(k)$, then system (1) can be also globally stabilized by the same controller $u(k)$. Thus, it remains to design a stabilizing controller $u(k)$ for (33), which can be stated as follows.

Step 1: Let $z(k)=\left[z_{1}^{\mathrm{T}}(k), z_{2}^{\mathrm{T}}(k)\right]^{\mathrm{T}}$ with $z_{1}=$ $\left[z_{11}^{\mathrm{T}}, z_{12}^{\mathrm{T}}, \ldots, z_{1 p_{1}}^{\mathrm{T}}\right]^{\mathrm{T}} \in \mathbf{R}^{2 p_{1} \times 1}$ and $z_{2}=\left[z_{21}, z_{22}, \ldots, z_{2 p_{2}}\right]^{\mathrm{T}} \in$ $\mathbf{R}^{p_{2} \times 1}$. We first consider the $z_{2}$-subsystem of (33), namely, $z_{2}(k+1)=A_{22} z_{2}(k)+b_{02} \sigma(u(k))$, which is in the form of system (8) with $n=p_{2}$. Then by using Theorem 2 , the stabilizing control can be designed as $u(k)=-u_{2 p_{2}}(k)$ with

$$
\left\{\begin{array}{l}
u_{2 i}(k)=\sigma_{e_{2 i}(k)}\left(\lambda_{i} z_{2 i}(k)+u_{2, i-1}(k)\right), i \in \mathbf{I}\left[2, p_{2}\right], \\
u_{21}(k)=\sigma_{e_{21}(k)}\left(\lambda_{1} z_{21}(k)-u_{1 p_{1}}(k)\right),
\end{array}\right.
$$

where $u_{1 p_{1}}(k)$ is to be designed later, and the parameters can be determined accordingly. Similar to the proof of Theorem 2, we conclude that there exists a finite number $K_{2} \geq 0$ such that the closed-loop system consisting of (33) and (35) can be simplified as, for all $k \geq K_{2}$,

$$
z(k+1)=\left[\begin{array}{ll}
A_{11} & \\
& \tilde{A}_{22}
\end{array}\right] z(k)+\left[\begin{array}{l}
b_{01} \\
b_{02}
\end{array}\right] u_{1 p_{1}}(k),
$$

in which all eigenvalues of $\tilde{A}_{22}$ are $1-\lambda_{i} \in(0,1), i \in \mathbf{I}\left[1, p_{2}\right]$, since $\lambda_{i} \in(0,1), i \in \mathbf{I}\left[1, p_{2}\right]$.

Step 2: We consider $z_{1}$-subsystem of (36), namely, $z_{1}(k+1)=$ $A_{11} z_{1}(k)+b_{01} u_{1 p_{1}}(k)$, which is in the form of (19) with $p=p_{1}$. 
Then by using Theorem 3, the subcontroller $u_{1 p_{1}}(k)$ can be designed as

$$
\left\{\begin{aligned}
u_{1 i}(k)= & \sigma_{\varepsilon_{i}}\left(F_{i} z_{1 i}(k)+u_{1, i-1}(k)\right), i \in \mathbf{I}\left[2, p_{1}\right], \\
u_{11}(k)= & \sigma_{\varepsilon_{1}}\left(F_{1} z_{11}(k)\right),
\end{aligned}\right.
$$

where the parameters can be determined accordingly. Similar to the proof of Theorem 3, we conclude that there exists a finite number $K_{1} \geq K_{2}$ such that the closed-loop system can be simplified as

$$
z(k+1)=\left[\begin{array}{cc}
\tilde{A}_{11} & \\
\tilde{A}_{21} & \tilde{A}_{22}
\end{array}\right] z(k), \forall k \geq K_{1},
$$

where $\tilde{A}_{11}$ and $\tilde{A}_{21}$ are some suitable matrices with $\left|\lambda\left(\tilde{A}_{11}\right)\right|<1$. As a result, system (38) is asymptotically stable.

Step 3: Combining (35) and (37) gives the global stabilizing controller $u(k)$ for system (1) with $m=1$, where $z(k)$ satisfies $z(k)=Q_{\mathrm{c}}\left(A_{\mathrm{t}}, B_{\mathrm{t}}\right) Q_{\mathrm{c}}^{-1}(A, B) y(k)$ with $y(k)$ given by $(2)$. The case by utilizing Theorem 1 can be considered similarly.

\section{B. Multi-input Case}

Under Assumption 1, when $m \geq 2$, system (3) can be further transformed into a series of linear systems with a single input by the Wonham canonical form decomposition [13], namely, there exists an invertible transformation $z=T y$ such that system (3) is transformed into

$$
z(k+1)=\bar{A} z(k)+\bar{B} \sigma(u(k)),
$$

where $(\bar{A}, \bar{B})$ is in the following form

$\bar{A}=\left[\begin{array}{cccc}\bar{A}_{1} & \bar{A}_{12} & \cdots & \bar{A}_{1 l} \\ & \bar{A}_{2} & \cdots & \bar{A}_{2 l} \\ & & \ddots & \vdots \\ & & & \bar{A}_{l}\end{array}\right], \bar{B}=\left[\begin{array}{ccccc}\bar{b}_{1} & & & & * \\ & \bar{b}_{2} & & & * \\ & & \ddots & & \vdots \\ & & & \bar{b}_{l} & *\end{array}\right]$

in which $\left(\bar{A}_{i}, \bar{b}_{i}\right) \in\left(\mathbf{R}^{n_{i} \times n_{i}}, \mathbf{R}^{n_{i} \times 1}\right), i \in \mathbf{I}[1, l]$, are all controllable and $l \leq m$. As each subsystem $\left(\bar{A}_{i}, \bar{b}_{i}\right)$ has a single input, we can utilize the proposed methods above to design the corresponding global stabilizing controller $u_{i}$. However, these $l$ subsystems are coupled with each other, which brings some difficulties in the design. To solve this problem, inspired by the treatment for the continuoustime case in [22], we give the design procedure in an explicit and recursive way as follows.

Step 1: We first consider the $l$-th subsystem of (39), namely, $z_{l}(k+1)=\bar{A}_{l} z_{l}(k)+\bar{b}_{l} \sigma\left(u_{l}(k)\right)$. which is a single-input system. Then by utilizing the design method in Section IV-A, a nonlinear controller $u_{l}=u_{l}\left(z_{l}\right)$ with $\left|u_{l}\right| \leq 1$ can be easily designed. Moreover, there exists a $K_{l}>0$ such that $u_{l}(k)=k_{l} z_{l}(k)$, $\forall k \geq K_{l}$. As a result, the closed-loop system can be simplified as $z_{l}(k+1)=\left(\bar{A}_{l}+\bar{b}_{l} k_{l}\right) z_{l}(k) \triangleq \bar{A}_{l}^{\mathrm{c}} z_{l}(k)$, where $\bar{A}_{l}^{\mathrm{c}}$ is Schur stable.

Step 2: We next consider the $(l-1)$-th subsystem of (39), namely,

$\left\{\begin{aligned} z_{l-1}(k+1) & =\bar{A}_{l-1} z_{l-1}(k)+\bar{A}_{l-1, l} z_{l}(k)+\bar{b}_{l-1} \sigma\left(u_{l-1}(k)\right) \\ z_{l}(k+1) & =\bar{A}_{l}^{\mathrm{c}} z_{l}(k) .\end{aligned}\right.$

Since $\lambda\left(\bar{A}_{l-1}\right) \cap \lambda\left(\bar{A}_{l}^{\mathrm{c}}\right)=\emptyset$, then the Sylvester matrix equation $\bar{A}_{l-1} T_{l-1, l}-T_{l-1, l} \bar{A}_{l}^{\mathrm{c}}=\bar{A}_{l-1, l}$ has the unique solution $T_{l-1, l}$. Now denote

$$
\left[\begin{array}{c}
w_{l-1} \\
w_{l}
\end{array}\right]=\left[\begin{array}{cc}
I_{n_{l-1}} & T_{l-1, l} \\
& I_{n_{l}}
\end{array}\right]\left[\begin{array}{c}
z_{l-1} \\
z_{l}
\end{array}\right],
$$

by which, one can easily verify that system (40) is algebraically equivalent to

$$
\left\{\begin{aligned}
w_{l-1}(k+1) & =\bar{A}_{l-1} w_{l-1}(k)+\bar{b}_{l-1} \sigma\left(u_{l-1}(k)\right), \\
w_{l}(k+1) & =\bar{A}_{l}^{\mathrm{c}} w_{l}(k) .
\end{aligned}\right.
$$

Again, by utilizing the design method in Section IV-A for the first subsystem in (42), a nonlinear controller $u_{l-1}=u_{l-1}\left(w_{l-1}\right)$ with $\left|u_{l-1}\right| \leq 1$ can be designed. Moreover, there exists a $K_{l-1}>K_{l}$ such that $u_{l-1}(k)=k_{l-1} w_{l-1}(k), \forall k \geq K_{l-1}$, where $w_{l-1}=$ $z_{l-1}+T_{l-1, l} z_{l}$, and $k_{l-1}$ is such that $\left(\bar{A}_{l-1}+\bar{b}_{l-1} k_{l-1}\right)$ is Schur

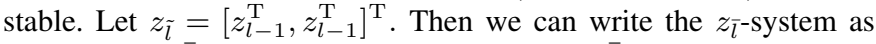
$z_{\tilde{l}}(k+1)=\bar{A}_{l-1}^{\mathrm{c}} z_{\tilde{l}}(k), \forall k \geq K_{l-1}$, where $\bar{A}_{l-1}^{\mathrm{c}}$ is Schur stable.

Step 3: We then consider the $(l-2)$-th subsystem of (39), namely,

$\left\{\begin{aligned} z_{l-2}(k+1) & =\bar{A}_{l-2} z_{l-2}(k)+\tilde{A}_{l-1, l} z_{\tilde{l}}(k)+\bar{b}_{l-2} \sigma\left(u_{l-2}(k)\right), \\ z_{\tilde{l}}(k+1) & =\bar{A}_{l-1}^{\mathrm{c}} z_{\tilde{l}}(k),\end{aligned}\right.$ which is exactly in the form of (40), where $\tilde{A}_{l-1, l}=$ $\left[\bar{A}_{l-2, l-1}, \bar{A}_{l-2, l}\right]$. Therefore, repeating the above process produces finally the controllers $u_{i}, i=l, l-1, \ldots, 1$.

\section{Multi-input-delay Case}

We finally mention that the proposed approaches can be extended to solve the global stabilization problem of general discrete-time linear systems with multiple input delays by bounded controls. A brief discussion is given as follows. Consider the discrete-time linear system with multiple input delays:

$$
x(k+1)=A x(k)+\sum_{i=1}^{p} B_{i} \sigma_{a_{i}}\left(u\left(k-r_{i}\right)\right),
$$

where $A \in \mathbf{R}^{n \times n}, B_{i} \in \mathbf{R}^{n \times m}, i \in \mathbf{I}[1, q], r_{i}, i \in$ $\mathbf{I}[1, q]$, are some positive integers denoting input delays, and $\sigma_{a_{i}}(u), i \in \mathbf{I}[1, q]$, are the vector-valued saturation functions with the saturation levels indicated respectively by the vectors $a_{i}=$ $\left[a_{i 1}, a_{i 2}, \ldots, a_{i m}\right]$ with $a_{i j}>0, i \in \mathbf{I}[1, q], j \in \mathbf{I}[1, m]$, namely, $\sigma_{a_{i}}(u)=\left[\sigma_{a_{i 1}}\left(u_{1}\right), \sigma_{a_{i 2}}\left(u_{2}\right), \ldots, \sigma_{a_{i m}}\left(u_{m}\right)\right]^{\mathrm{T}}$, in which $u=\left[u_{1}, u_{2}, \ldots, a_{m}\right]^{\mathrm{T}}$. Without loss of generality, we impose the following assumption on system (43).

Assumption 2: All eigenvalues of $A$ are on the unit circle and the matrix pair $(A, B)$ is controllable, where

$$
B=A^{-r_{1}} B_{1}+A^{-r_{2}} B_{2}+\ldots+A^{-r_{p}} B_{p} .
$$

Consider the following new state vector:

$$
y(k)=x(k)+\sum_{i=1}^{p} \sum_{j=k}^{k+r_{j}-1} A^{k-j-1} B_{i} \sigma_{a_{i}}\left(u\left(j-r_{i}\right)\right) .
$$

Then by using (45), system (43) can be expressed as

$$
y(k+1)=A y(k)+\sum_{i=1}^{p} A^{-r_{i}} B_{i} \sigma_{a_{i}}(u(k)) .
$$

Let $a=\left[\min _{j \in \mathbf{I}[1, m]}\left\{a_{1 j}\right\}, \ldots, \min _{j \in \mathbf{I}[1, m]}\left\{a_{p j}\right\}\right]^{\mathrm{T}}$, and define $u=\sigma_{a}(v)$. Then system (46) can be continued as $y(k+1)=$ $A y(k)+\sum_{i=1}^{p} A^{-r_{i}} B_{i} \sigma_{a}(v(k))=A y(k)+B \sigma_{a}(v(k))$, where we have noticed (44), which has the same form as (3), and then the rest is similar to the above analysis for discrete-time systems with a single input delay.

\section{A. A Chain of Integrators}

In this subsection, we use the 4-th order discrete-time multiple integrators in the form of (4) to illustrate the proposed methods in Section III-A. For saving space, here we just take Theorem 2 as an example. Let $r=2$. For convenience, we define

$$
T_{1}=\left[\begin{array}{cccc}
\omega_{11} & \omega_{12} & \omega_{13} & 1 \\
0 & \omega_{22} & \omega_{23} & 1 \\
0 & 0 & \omega_{33} & 1 \\
0 & 0 & 0 & 1
\end{array}\right]
$$




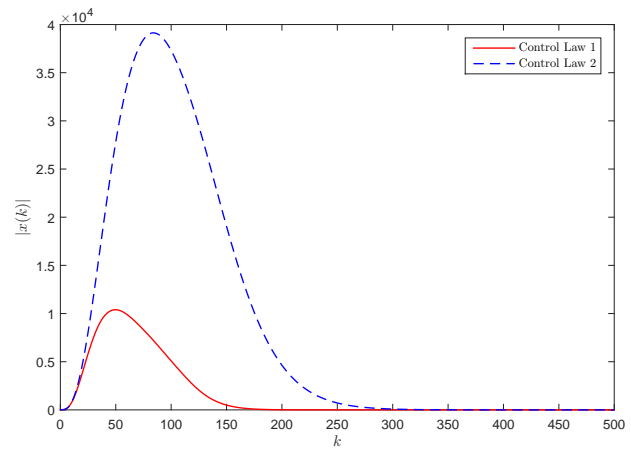

Fig. 1. Comparison of trajectory of 4-th order chain of integrators with different controllers.

where $\omega_{11}=\lambda_{2} \lambda_{3} \lambda_{4}, \omega_{12}=\lambda_{2} \lambda_{3}+\lambda_{2} \lambda_{4}+\lambda_{3} \lambda_{4}, \omega_{13}=\lambda_{2}+$ $\lambda_{3}+\lambda_{4}, \omega_{22}=\lambda_{3} \lambda_{4}, \omega_{23}=\lambda_{3}+\lambda_{4}, \omega_{33}=\lambda_{4}$, and $\lambda_{i}, i \in \mathbf{I}[2,4]$, are some given positive scalars. Consider the following two control design methods.

- Control Law 1: This control law is based on Theorem 2 and takes the form $u_{1}=-\sigma_{e_{4}}\left(\lambda_{4} z_{4}+\sigma_{e_{3}}\left(\lambda_{3} z_{3}+\sigma_{e_{2}}\left(\lambda_{2} z_{2}+\right.\right.\right.$ $\left.\left.\left.\sigma_{e_{1}}\left(\lambda_{1} z_{1}\right)\right)\right)\right)$, where $\lambda_{i}, i \in \mathbf{I}[1,4]$, satisfy (11), $z=$ $\left[z_{1}, z_{2}, z_{3}, z_{4}\right]^{\mathrm{T}}$ satisfies $z=T_{1}\left(A_{\mathrm{s}}^{2} x(k)+b_{\mathrm{s}} \sigma\left(u_{1}(k-1)\right)+\right.$ $\left.A_{\mathrm{s}} b_{\mathrm{s}} \sigma\left(u_{1}(k-2)\right)\right)$, in which $\left(A_{\mathrm{s}}, b_{\mathrm{s}}\right)$ is in the form of (5) with $n=4, e_{i}, i \in \mathbf{I}[1,4]$, satisfy (14) with $\varepsilon_{i}, i \in \mathbf{I}[1,4]$, satisfying (13). By Theorem 2, we choose $\varepsilon_{4}=1, \varepsilon_{3}=\eta / 2$, $\varepsilon_{2}=\eta^{2} / 4$ and $\varepsilon_{1}=\eta^{3} / 8$ with $\eta=0.99$.

- Control Law 2: This control law is based on Theorem 4 in [24] and takes the form $u_{2}=$ $-\sigma_{\varepsilon_{4}}\left(\lambda_{4} y_{4}+\sigma_{\varepsilon_{3}}\left(\lambda_{3} y_{3}+\sigma_{\varepsilon_{2}}\left(\lambda_{2} y_{2}+\sigma_{\varepsilon_{1}}\left(\lambda_{1} y_{1}\right)\right)\right)\right)$, where $\left\{y_{i}, \varepsilon_{i}, \lambda_{i}\right\}, i \in \mathbf{I}[1,4]$, are the same with Control Law 7 in [24].

We first determine the parameters $\lambda_{i}, i \in \mathbf{I}[1,4]$, in Control Laws 1-2 to achieve the best control performance. Let $\lambda_{i}=\lambda, i \in \mathbf{I}[1,4]$. For the given initial condition $x(0)=[-5,-5,5,5]^{\mathrm{T}}$ and $u(\theta)=$ $0, \theta \in \mathbf{I}[-2,-1]$, simulation results show that, $\lambda=0.1$ for Control Law 1 and $\lambda=0.05$ for Control Law 2 lead to the best control performance, respectively.

Finally, we give a comparison among Control Laws 1-2. Let $\lambda_{i}=0.1, i \in \mathbf{I}[1,4]$, in Control Law 1 and $\lambda_{i}=0.05, i \in \mathbf{I}[1,4]$, in Control Law 2. The resulting 2-norm of the state trajectories of the closed-loop systems are recorded in Fig. 1. It follows that Control Law 1 established in this paper outperforms Control Law 2 as discussed in Remark 3.

\section{B. A Chain of Oscillators}

To illustrate Theorem 3, we consider discrete-time linear system (15) with $r=2$, and

$$
A_{\mathrm{\circ}}=\left[\begin{array}{cccc}
\frac{\sqrt{2}}{2} & -\frac{\sqrt{2}}{\sqrt{2}} & 0 & 0 \\
\frac{\sqrt{2}}{2} & \frac{\sqrt{2}}{2} & 1 & 0 \\
0 & 0 & 0 & -1 \\
0 & 0 & 1 & 0
\end{array}\right], b_{\mathrm{o}}=\left[\begin{array}{l}
0 \\
0 \\
0 \\
1
\end{array}\right] .
$$

It follows that $\lambda\left(A_{\circ}\right)=\left\{\frac{\sqrt{2}}{2} \pm \frac{\sqrt{2}}{2} \mathrm{j}, \pm \mathrm{j}\right\}$ and $\left(A_{\circ}, b_{\mathrm{o}}\right)$ is controllable. Let

$$
T_{2}=\left[\begin{array}{cccc}
\frac{\gamma_{2}}{2}-1 & 1 & \frac{\sqrt{2}}{2} & 0 \\
-1 & \frac{\gamma_{2}}{2}-1 & -\frac{\sqrt{2}}{2} & 1 \\
0 & 0 & 1 & 0 \\
0 & 0 & 0 & 1
\end{array}\right],
$$
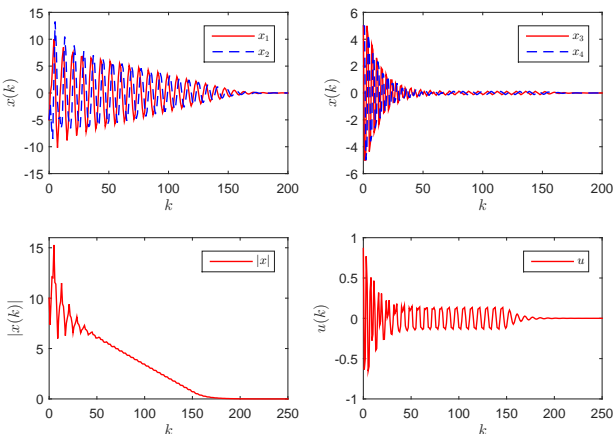

Fig. 2. State and control signals of the closed-loop system under controller (50).

in which $\gamma_{2} \in(0,2]$. Based on Theorem 3, the corresponding stabilizing controller can be designed as:

$$
u_{3}=\sigma_{\varepsilon_{2}}\left(-\frac{\gamma_{2}}{2} z_{3}+\sigma_{\varepsilon_{1}}\left(-\frac{\sqrt{2} \gamma_{1}}{4} z_{2}-\frac{\sqrt{2} \gamma_{1}}{4} z_{1}\right)\right)
$$

where $z=\left[z_{1}, z_{2}, z_{3}, z_{4}\right]^{\mathrm{T}}$ satisfies $z=$ $T_{2}\left(A_{\mathrm{o}}^{2} x(k)+b_{\mathrm{o}} \sigma\left(u_{3}(k-1)\right)+A_{\mathrm{o}} b_{\mathrm{o}} \sigma\left(u_{3}(k-2)\right)\right)$. By Theorem 3 , we can choose $\gamma_{1}=\gamma_{2}=0.3, \lambda_{2}=0.93, \eta_{2}=0.001$, $\mu=0.99, P_{2}=[6.2072,0 ; 0,7.2072], p_{2}^{+}=7.2072$ and $p_{2}^{-}=6.2072$. Let the initial condition be $x(0)=[-5,-5,5,5]^{\mathrm{T}}$ and $u(\theta)=0, \forall \theta \in \mathbf{I}[-2,-1]$. The state and control signals the closed-loop system are recorded in Fig. 2. It follows that the states converge to the origin, which indicates asymptotic stability of the closed-loop system.

\section{A General Linear System}

In this subsection, we consider the following discrete-time system:

$$
x(k+1)=A x(k)+b \sigma(u(k-2)),
$$

where

$$
A=\left[\begin{array}{ccccc}
0 & -1 & 0 & 0 & 0 \\
1 & 0 & 1 & 0 & 0 \\
0 & 0 & 1 & 1 & 0 \\
0 & 0 & 0 & 1 & 1 \\
0 & 0 & 0 & 0 & 1
\end{array}\right], b=\left[\begin{array}{l}
0 \\
0 \\
0 \\
0 \\
1
\end{array}\right]
$$

It follows that $\lambda(A)=\{1,1,1, \pm \mathrm{j}\}$ and $(A, b)$ is controllable. Let

$$
A_{\mathrm{t}}=\left[\begin{array}{ccccc}
0 & -1 & 0 & 0 & 0 \\
1 & 0 & \lambda_{1} & \lambda_{2} & \lambda_{3} \\
0 & 0 & 1 & \lambda_{2} & \lambda_{3} \\
0 & 0 & 0 & 1 & \lambda_{3} \\
0 & 0 & 0 & 0 & 1
\end{array}\right], b_{\mathrm{t}}=\left[\begin{array}{l}
0 \\
1 \\
1 \\
1 \\
1
\end{array}\right],
$$

and $T_{3}=Q_{\mathrm{c}}\left(A_{\mathrm{t}}, b_{\mathrm{t}}\right) Q_{\mathrm{c}}^{-1}(A, b)$. Then combining Theorems 2-3 (Theorem 1 is also valid, which is omitted for brevity), the corresponding stabilizing controller for (51) can be designed as

$$
u_{4}=-\sigma_{e_{4}}\left(\lambda_{3} z_{5}+\sigma_{e_{3}}\left(\lambda_{2} z_{4}+\sigma_{e_{2}}\left(\lambda_{1} z_{3}-\sigma_{\varepsilon_{1}}\left(-\frac{\gamma}{2} z_{1}\right)\right)\right)\right)_{(53)} \text {, }
$$

where $z=\left[z_{1}, z_{2}, z_{3}, z_{4}, z_{5}\right]^{\mathrm{T}}$ satisfies $z=T_{3}\left(A^{2} x(k)+b \sigma\left(u_{4}(k-\right.\right.$ $\left.1))+A b \sigma\left(u_{4}(k-2)\right)\right)$ with $(A, b)$ satisfying (52), $e_{4}=\varepsilon_{4}, e_{1}=\varepsilon_{1}$, and for $i \in \mathbf{I}[3,4], e_{i-1}=e_{i}-\left|\lambda_{i} z_{i}\right|$, if $\left|\lambda_{i} z_{i}\right| \leq \varepsilon_{i}-\varepsilon_{i-1}$, and $e_{i-1}=\varepsilon_{i-1}$. By Theorems 2--3, we can choose $\lambda_{i}=0.15, i \in$ $\mathbf{I}[1,3], \gamma=0.2, \varepsilon_{4}=1, \varepsilon_{3}=\eta / 2, \varepsilon_{2}=\eta^{2} / 4$ and $\varepsilon_{1}=\eta^{3} / 8$ with $\eta=0.99$. Let the initial condition be $x(0)=[-5,-5,5,5,5]^{\mathrm{T}}$ and $u(\theta)=0, \forall \theta \in \mathbf{I}[-2,-1]$. The state and control signals of the closed-loop system are recorded in Fig. 3. It follows that the states 

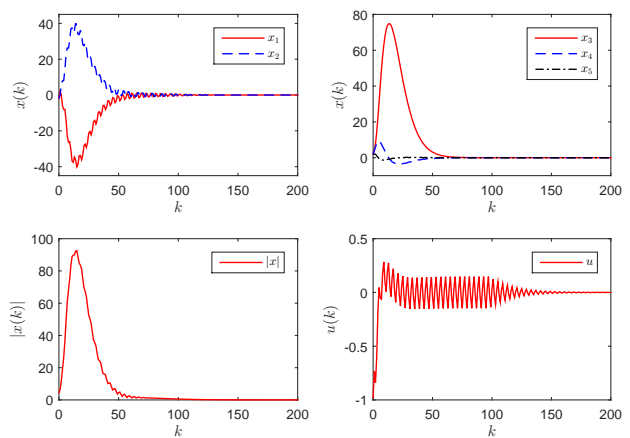

Fig. 3. State and control signals of the closed-loop system consisting of (51) and (53).

converge to the origin, which indicates asymptotic stability of the closed-loop system.

\section{Conclusion}

This paper has investigated the global stabilization of discretetime time-delay linear systems with bounded controls. By utilizing prediction technique, the considered time-delay system was first transformed into a delay-free system. Then, by further utilizing saturation functions technique, nonlinear controllers, which use both current and delayed feedback information, have been established for global stabilizing systems characterized by both a chain of discretetime integrators and oscillators, and explicit conditions to guarantee the stability of closed-loop systems have been given. Based on the above results, a recursive design procedure for general discrete-time linear systems with multiple inputs and/or multiple input delays has also been proposed. Finally, the effectiveness and superiority of the developed methods in this paper have been illustrated by the simulation results.

\section{APPENDIX}

\section{Proof of Lemma 2}

By using Proposition 1 in [21], we only need to show that $\left(A_{\mathrm{ot}}, b_{\mathrm{ot}}\right)$ is controllable since $\lambda\left(A_{\mathrm{ot}}\right)=\lambda\left(A_{\mathrm{o}}\right)$. It follows from Lemma 3 that $A_{\mathrm{c} i}, i \in \mathbf{I}[1, p]$ (see (27)) are all asymptotically stable, namely, $\left|\lambda\left(A_{\mathrm{c} i}\right)\right|<1, i \in \mathbf{I}[1, p]$, which implies that $\lambda\left(A_{\mathrm{c} i}\right) \cap \lambda\left(A_{j}\right)=\emptyset$, $j \in \mathbf{I}[1, i-1], i \in \mathbf{I}[2, p]$. Moreover, one can verify that $\left(A_{i}, b\right)$, $i \in \mathbf{I}[1, p]$, are all controllable. Thus, by using Proposition 2 in [22], we conclude that $\left(A_{\mathrm{ot}}, b_{\mathrm{ot}}\right)$ is controllable. The proof is finished.

\section{Proof of Lemma 3}

It follows from (24) that, for any $i \in \mathbf{I}[1, n-1], P A^{i}=$ $A^{\mathrm{T}} P A^{i+1}=\left(A^{\mathrm{T}}\right)^{2} P A^{i+2}=\cdots=\left(A^{\mathrm{T}}\right)^{n-i} P A^{n}$, by which and (25), we can obtain

$$
\begin{aligned}
Q_{\mathrm{o}}(A, F) & \triangleq\left[\begin{array}{llll}
F^{\mathrm{T}} & A^{\mathrm{T}} F^{\mathrm{T}} & \cdots & \left(A^{\mathrm{T}}\right)^{n-1} F^{\mathrm{T}}
\end{array}\right]^{\mathrm{T}} \\
& =\operatorname{diag}\left\{D_{1}, D_{1}, \ldots, D_{1}\right\} D_{2} P A^{n}
\end{aligned}
$$

where $D_{1}=-\gamma\left(I+B^{\mathrm{T}} P B\right)^{-1}$ and $D_{2}=$ $\left[A^{n-1} B, A^{n-2} B, \ldots, A B, B\right]^{\mathrm{T}}$. Since $(A, B)$ is controllable and $\operatorname{det}(A) \neq 0$, it follows from (54) that $(A, F)$ is observable, by which we also know that $\left(A_{\mathrm{c}}, F\right)$ is observable. Moreover, if $\gamma \in(0,2]$, by using (24) and (25), we have

$$
A_{\mathrm{c}}^{\mathrm{T}} P A_{\mathrm{c}}-P=A^{\mathrm{T}} P A-P+2 A^{\mathrm{T}} P B F+F^{\mathrm{T}} B^{\mathrm{T}} P B F
$$

$$
\begin{aligned}
& =F^{\mathrm{T}}\left(-\frac{2}{\gamma} I+\left(-\frac{2}{\gamma}+1\right) B^{\mathrm{T}} P B\right) F \\
& \leq-\frac{2}{\gamma} F^{\mathrm{T}} F,
\end{aligned}
$$

which implies that $A_{\mathrm{c}}$ is asymptotically stable since $\left(A_{\mathrm{c}}, F\right)$ is observable and $P>0$. The rest of the proof is trivial and omitted.

\section{Proof of Theorem 3}

Before presenting the proof, we need the following preliminary results. Let $\alpha$ and $\beta$ be two real constants satisfying $\alpha^{2}+\beta^{2}=1$, $\beta \neq 0, \gamma \in(0,2],\left\{F_{\gamma}, A_{\varpi}, b\right\}$ be in the forms of (21)-(22) with $\left\{\gamma_{i}, \alpha_{i}, \beta_{i}\right\}$ being replaced by $\{\gamma, \alpha, \beta\}$, and $A_{\varpi c}=A_{\varpi}+b F_{\gamma}$. It follows from Lemma 3 that $A_{\varpi \mathrm{c}}$ is asymptotically stable, and there exist a $P_{\mathrm{c}}>0$ and a $\lambda \in(0,1)$ such that $A_{\varpi \mathrm{c}}^{\mathrm{T}} P_{\mathrm{c}} A_{\varpi \mathrm{c}} \leq \lambda^{2} P_{\mathrm{c}}$. Denote $p^{-}=\lambda_{\min }\left(P_{\mathrm{c}}\right)$ and $p^{+}=\lambda_{\max }\left(P_{\mathrm{c}}\right)$. Then we have the following lemma, whose proof will be given later.

Lemma 4: Let $\left\{A_{\varpi}, A_{\varpi \mathrm{c}}, F_{\gamma}, P_{\mathrm{c}}, b, \gamma, \lambda, p^{-}, p^{+}\right\}$be defined above. Consider the following planar nonlinear system

$$
\left\{\begin{aligned}
\varpi(k+1) & =A_{\varpi} \varpi(k)+b u(k), \\
u(k) & =\sigma_{\varepsilon_{2}}\left(F_{\gamma} \varpi(k)+\sigma_{\varepsilon_{1}}(d(k))\right),
\end{aligned}\right.
$$

where $\varepsilon_{1} \geq 0, \varepsilon_{2}>0$ are given scalars, $d$ is an external signal. If

$$
\left(\frac{3 \gamma p^{+}}{2(1-(1+\eta) \lambda) p^{-}}+1\right) \varepsilon_{1}<\varepsilon_{2}
$$

where $\eta \in(0,(1-\lambda) / \lambda)$, then there exists a finite number $K>0$ such that for all $k \geq K$, (55) can be simplified as

$$
\left\{\begin{aligned}
\varpi(k+1) & =\left(A_{\varpi}+b F_{\gamma}\right) \varpi(k)+b \sigma_{\varepsilon_{1}}(d(k)), \\
u(k) & =F_{\gamma} \varpi(k)+\sigma_{\varepsilon_{1}}(d(k)) .
\end{aligned}\right.
$$

Now we are ready to give the detailed proof of Theorem 3 . We first consider the $p$-th subsystem of (19), namely,

$$
\left\{\begin{aligned}
z_{p}(k+1) & =A_{p} z_{p}(k)+b u_{p}(k), \\
u_{p}(k) & =\sigma_{\varepsilon_{p}}\left(F_{p} z_{p}(k)+u_{p-1}(k)\right),
\end{aligned}\right.
$$

which is exactly in the form of (55) where we have noticed that $\sigma\left(u_{p}\right)=u_{p}$ by $\varepsilon_{p} \leq 1$. Notice that from (31), we have $\left|u_{i}(k)\right| \leq \varepsilon_{i}$, $i \in \mathbf{I}[1, p]$. Thus, by using Lemma 4 , if (30) with $i=p$ holds true, there exists a finite number $K_{p}>0$ such that, $u_{p}(k)=F_{p} z_{p}(k)+$ $u_{p-1}(k), \forall k \geq K_{p}$. As a result, system (19) reduces to

$$
\left\{\begin{aligned}
z_{1}(k+1)= & A_{1} z_{1}(k)-\sum_{i=2}^{p-1} b F_{i} z_{i}(k)+b u_{p-1}(k), \\
\vdots & \\
z_{p-1}(k+1)= & A_{p-1} z_{p-1}(k)+b u_{p-1}(k), \\
z_{p}(k+1)= & A_{\mathrm{c} p} z_{p}(k)+b u_{p-1}(k),
\end{aligned}\right.
$$

where $u_{p-1}(k)$ satisfies (31). Repeat the above process with the subcontrollers $u_{i}(k), i=p-1, p-2, \ldots, 1$, we know that there exists a finite number $K_{1}>K_{2}$ such that, for all $k \geq K_{1}$, there holds

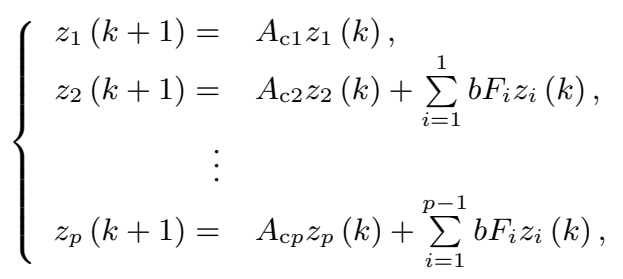

which is exponentially stable if and only if the set of planar systems $\varphi_{i}(k+1)=A_{\mathrm{c} i} \varphi_{i}(k), i \in \mathbf{I}[1, p]$, with $A_{\mathrm{c} i}, i \in \mathbf{I}[1, p]$, satisfying (27), are all exponentially stable. This is obvious by Lemma 3 since $\gamma_{i} \in(0,2], i \in \mathbf{I}[1, p]$. The proof is finished. 


\section{Proof of Lemma 4}

Let $\tilde{\varpi}=F_{\gamma} \varpi+\sigma_{\varepsilon_{1}}(d)$, which is a scalar. Then system (55) can be rewritten as

$$
\varpi(k+1)=A_{\varpi} \varpi(k)+b \sigma_{\varepsilon_{2}}(\tilde{\varpi}(k)) .
$$

Now we choose the Lyapunov function $V_{0}(\varpi)=\varpi^{\mathrm{T}} \varpi$, whose timeshift along the trajectories of system (59) satisfies

$$
\begin{aligned}
\nabla V_{0}= & \left(A_{\varpi} \varpi+b \sigma_{\varepsilon_{2}}(\tilde{\varpi})\right)^{\mathrm{T}}\left(A_{\varpi} \varpi+b \sigma_{\varepsilon_{2}}(\tilde{\varpi})\right)-\varpi^{\mathrm{T}} \varpi \\
= & 2 \varpi^{\mathrm{T}} A_{\varpi}^{\mathrm{T}} b \sigma_{\varepsilon_{2}}(\tilde{\varpi})+\sigma_{\varepsilon_{2}}^{2}(\tilde{\varpi}) \\
= & -\frac{2}{\gamma} \tilde{\varpi} \sigma_{\varepsilon_{2}}(\tilde{\varpi})+\left(-\frac{2}{\gamma} \tilde{\varpi}+\sigma_{\varepsilon_{2}}(\tilde{\varpi})\right) \sigma_{\varepsilon_{2}}(\tilde{\varpi}) \\
& +\frac{4}{\gamma} \sigma_{\varepsilon_{1}}(d) \sigma_{\varepsilon_{2}}(\tilde{\varpi}) \\
\leq & -\frac{2}{\gamma} \tilde{\varpi} \sigma_{\varepsilon_{2}}(\tilde{\varpi})+\frac{4}{\gamma} \varepsilon_{1} \varepsilon_{2},
\end{aligned}
$$

where we have noticed that $A_{\varpi}^{\mathrm{T}} A_{\varpi}-I_{2}=0, b^{\mathrm{T}} b=1, \varpi^{\mathrm{T}} A_{\varpi}^{\mathrm{T}} b=$ $-2\left(\tilde{\varpi}-\sigma_{\varepsilon_{1}}(d)\right) / \gamma$ and $\left(-2 \tilde{\varpi} / \gamma+\sigma_{\varepsilon_{2}}(\tilde{\varpi})\right) \sigma_{\varepsilon_{2}}(\tilde{\varpi}) \leq 0$. Rewrite system (59) as

$$
\varpi(k+1)=A_{\varpi \mathrm{c}} \varpi(k)+b\left(\sigma_{\varepsilon_{1}}(d(k))-\tilde{\varpi}(k)+\sigma_{\varepsilon_{2}}(\tilde{\varpi}(k))\right) .
$$

We choose $V_{1}(\varpi)=\left|P_{\mathrm{c}}^{1 / 2} \varpi\right|$ where $P_{\mathrm{c}}^{1 / 2}$ denotes the unique symmetric positive definite matrix $X$ satisfying $X^{2}=P_{\mathrm{c}}$. The timeshift of $V_{1}(\varpi)$ along system (61) gives

$$
\begin{aligned}
\nabla V_{1} & =\left|P_{\mathrm{c}}^{\frac{1}{2}}\left(A_{\varpi \mathrm{c}} \varpi+b\left(\sigma_{\varepsilon_{1}}(d)-\tilde{\varpi}+\sigma_{\varepsilon_{2}}(\tilde{\varpi})\right)\right)\right|-\left|P_{\mathrm{c}}^{\frac{1}{2}} \varpi\right| \\
& \leq-(1-\lambda)\left|P_{\mathrm{c}}^{\frac{1}{2}} \varpi\right|+\left|P_{\mathrm{c}}^{\frac{1}{2}}\right|\left|\tilde{\varpi}-\sigma_{\varepsilon_{2}}(\tilde{\varpi})\right|+\left|P_{\mathrm{c}}^{\frac{1}{2}}\right| \varepsilon_{1},
\end{aligned}
$$

where we have noticed that $A_{\varpi \mathrm{c}}^{\mathrm{T}} P_{\mathrm{c}} A_{\varpi \mathrm{c}} \leq \lambda^{2} P_{\mathrm{c}}$ and $|b|=1$. On the other hand, for any $\tilde{\varpi} \in \mathbf{R}$, there holds $|\tilde{\varpi}-\sigma(\tilde{\varpi})| \leq \tilde{\varpi} \sigma(\tilde{\varpi})$ [10]. Hence, for any $\varepsilon_{2}>0$ and $\tilde{\varpi} \in \mathbf{R},\left|\tilde{\varpi}-\sigma_{\varepsilon_{2}}(\tilde{\varpi})\right| \leq \tilde{\varpi} \sigma_{\varepsilon_{2}}(\tilde{\varpi}) / \varepsilon_{2}$, by which inequality (62) can be continued as

$$
\nabla V_{1} \leq-(1-\lambda)\left|P_{\mathrm{c}}^{\frac{1}{2}} \varpi\right|+\frac{1}{\varepsilon_{2}}\left|P_{\mathrm{c}}^{\frac{1}{2}}\right| \tilde{\varpi} \sigma_{\varepsilon_{2}}(\tilde{\varpi})+\left|P_{\mathrm{c}}^{\frac{1}{2}}\right| \varepsilon_{1} .
$$

Now consider the Lyapunov function $V(\varpi)=\frac{\gamma}{2 \varepsilon_{2}}\left|P_{\mathrm{c}}^{1 / 2}\right| V_{0}(\varpi)+$ $V_{1}(\varpi)$, and define two class $\mathcal{K}$ functions $\pi_{1}$ and $\pi_{2}$ as $\pi_{1}(|\varpi|)=$ $\frac{\gamma}{2 \varepsilon_{2}}\left|P_{\mathrm{c}}^{1 / 2}\right||\varpi|^{2}+\sqrt{p^{-}}|\varpi|$ and $\pi_{2}(|\varpi|)=\frac{\gamma}{2 \varepsilon_{2}}\left|P_{\mathrm{c}}^{1 / 2}\right||\varpi|^{2}+$ $\sqrt{p^{+}}|\varpi|$. Obviously, there holds $\pi_{1}(|\varpi|) \leq V(\varpi) \leq \pi_{2}(|\varpi|)$ and $\pi_{1}^{-1}\left(\pi_{2}(|\varpi|)\right) \leq \sqrt{p^{+}}|\varpi| / \sqrt{p^{-}}$. By (60) and (63), the time-shift of $V(\varpi)$ along the trajectories of system (59) or (61) satisfies

$$
\begin{aligned}
\nabla V \leq & -(1-\lambda)\left|P_{\mathrm{c}}^{\frac{1}{2}} \varpi\right|+3\left|P_{\mathrm{c}}^{\frac{1}{2}}\right| \varepsilon_{1} \\
\leq & -(1-\lambda) \sqrt{p^{-}}|\varpi|+3 \sqrt{p^{+}} \varepsilon_{1} \\
\triangleq & -\eta \lambda \sqrt{p^{-}}|\varpi|-(1-(1+\eta) \lambda) \sqrt{p^{-}}|\varpi| \\
& +3 \sqrt{p^{+}} \varepsilon_{1},
\end{aligned}
$$

where $\eta \in\left(0, \frac{1-\lambda}{\lambda}\right)$. When $-(1-(1+\eta) \lambda) \sqrt{p^{-}}|\varpi|+3 \sqrt{p^{+}} \varepsilon_{1} \leq 0$, namely, $|\varpi| \geq 3 \sqrt{p^{+}} \varepsilon_{1} /\left((1-(1+\eta) \lambda) \sqrt{p^{-}}\right)$, it follows from (64) that $\nabla V(\varpi) \leq-\eta \lambda \sqrt{p^{-}}|\varpi|$. Then, parallel to Theorem 4.18 in [4], we can conclude that the trajectory of closed-loop system (55) will enter the set $\left\{|\varpi| \leq r_{\mathrm{d}}\right\}$ with

$r_{\mathrm{d}} \leq \pi_{1}^{-1}\left(\pi_{2}\left(\frac{3 \sqrt{p^{+}} \varepsilon_{1}}{(1-(1+\eta) \lambda) \sqrt{p^{-}}}\right)\right) \leq \frac{3 p^{+} \varepsilon_{1}}{(1-(1+\eta) \lambda) p^{-}}$,

after a finite number $K \geq 0$ and remains therein for all future time. As a result, we have $\left|F_{\gamma} \varpi+\sigma_{\varepsilon_{1}}(d)\right| \leq \gamma|\varpi| / 2+\varepsilon_{1}=$ $\left(3 \gamma p^{+} /\left(2(1-(1+\eta) \lambda) p^{-}\right)+1\right) \varepsilon_{1} \leq \varepsilon_{2}, \forall k \geq K$, where the last inequality is guaranteed by (56). Therefore, the system and control in (55) can be naturally simplified as (57) for all $k \geq K$. The proof is finished.

\section{REFERENCES}

[1] S. Bittanti and P. Colaneri, Periodic systems: filtering and control, Springer Science \& Business Media, 2009.

[2] A. Gonzalez, P. Garcia, P. Albertos, P. Castillo, and R. Lozano, "Robustness of a discrete-time predictor-based controller for time-varying measurement delay," Control Eng. Pract., vol. 20, pp. 102-110, 2012.

[3] G. Kaliora and A. Astolfi, "Nonlinear control of feedforward systems with bounded signals," IEEE Trans. Autom. Control, vol. 49, pp. 1975 1990, 2004.

[4] H. K. Khalil, Nonlinear Systems. Upper Saddle River NJ: Prentice Hall, 2002.

[5] N. Marchand and A. Hably, "Global stabilization of multiple integrators with bounded controls," Automatica, vol. 41, pp. 2147-2152, 2005.

[6] N. Marchand, A. Hably, and A. Chemori, "Global stabilization with low computational cost of the discrete-time chain of integrators by means of bounded controls," IEEE Trans. Autom. Control, vol. 52, pp. 948-952, 2007.

[7] F. Mazenc, S. Mondié, and S.-I. Niculescu, "Global asymptotic stabilization for chains of integrators with a delay in the input," IEEE Trans. Autom. Control, vol. 48, pp. 57-63, 2003.

[8] F. Mazenc, S. Mondié, and R. Francisco, "Global asymptotic stabilization of feedforward systems with delay in the input," IEEE Trans. Autom. Control, vol. 49, pp. 844-850, 2004.

[9] F. Mazenc and H. Nijmeijer, "Forwarding in discrete-time nonlinear systems," Int. J. Control, vol. 71, pp. 823-835, 1998.

[10] H. J. Sussmann, E. D. Sontag, and Y. D. Yang, "A general result on the stabilization of linear systems using bounded controls," IEEE Trans. Autom. Control, vol. 39, pp. 2411-2425, 1994.

[11] H. J. Sussmann and Y. Yang, On the stabilizability of multiple integrators by means of bounded feedback controls, Proceedings of the 30nd IEEE Conference on Decision and Control, pp. 70-72, 1991.

[12] A. R. Teel, "Global stabilization and restricted tracking for multiple integrators with bounded controls," Syst. Control Lett., vol. 18, pp. 165 171, 1992.

[13] W.M. Wonham, "On pole assignment in multi-input controllable linear systems," IEEE Trans. Autom. Control, vol. 12, no. 6, pp. 660-665, 1967.

[14] Y. Xie and Z. Lin, "Global stabilisation of discrete-time linear systems using event-triggered bounded controls," IET Control Theory Appl., vol. 13, pp. 1355-1366, 2018

[15] K. Yakoubi and Y. Chitour, "Linear systems subject to input saturation and time delay: Global asymptotic stabilization," IEEE Trans. Autom. Control, vol. 52, pp. 874-879, 2007.

[16] K. Yakoubi and Y. Chitour, "On the stabilization of linear discretetime delay systems subject to input saturation," Advanced Strategies in Control Systems with Input and Output Constraints, Springer Berlin Heidelberg, 2007.

[17] X. Yang, B. Zhou, and J. Lam, "Global stabilization of multiple oscillator systems by delayed and bounded feedback," IEEE Trans. Circuits Syst. II: Exp. Briefs, vol. 64, pp. 675-679, 2017.

[18] Y. D. Yang, E. D. Sontag, and H. J. Sussmann, "Global stabilization of linear discrete-time systems with bounded feedback," Syst. Control Lett., vol. 30, pp. 273-281, 1997.

[19] H. Ye, "Global stabilisation of complicated feedforward non-linear systems by constructing state-dependent saturation levels," IET Control Theory Appl., vol. 10, pp. 2071-2082, 2016.

[20] H. Ye, "Stabilization of uncertain feedforward nonlinear systems with application to under-actuated systems," IEEE Trans. Autom. Control, vol. 64, pp. 3484-3491, 2019.

[21] B. Zhou and G.-R. Duan, Global stabilisation of multiple integrators via saturated controls. IET Control Theory \& Applications, vol. 1, pp. 1586-1593, 2007.

[22] B. Zhou and Y. Liu, "Global stabilization of linear systems with bounded controls by the energy function with applications to the spacecraft rendezvous," Proc. 2017 Chin. Autom. Congress, pp. 2428-2435, 2017.

[23] B. Zhou and X. Yang, "Global stabilization of the multiple integrators system by delayed and bounded controls," IEEE Trans. Autom. Control, vol. 61, pp. 4222-4228, 2016.

[24] B. Zhou and X. Yang, "Global stabilization of discrete-time multiple integrators with bounded and delayed feedback," Automatica, vol. 97, pp. 306-315, 2018. 